\title{
Healing-Promoting Action of Dried Aluminium Hydroxide Gel Evaluated by the Method of Histological Measurements in Acetic Acid Ulcer of Rats
}

\author{
Mikio ITO, Akira FUJII and Yoshio SUZUKI \\ Department of Pharmacology, Faculty of Pharmacy. Meijo University \\ Tenpaku-ku. Nagoya 468, Japan \\ Accepted February 6, 1985
}

\begin{abstract}
The effects of dried aluminium hydroxide gel on the healing of rat acetic acid ulcer were studied in comparison to those of propantheline bromide by the method of histological measurements. Dried aluminium hydroxide gel not only decreased the macroscopic ulcer index and the defective area in the ulcerated region but also increased the decreasing index of exposed ulcer floor and the mucosal regeneration index without affecting the thickness of the ulcer base and the development index of collagen fibers. However, propantheline bromide did not have any beneficial effects.
\end{abstract}

Of the antiulcer agents, antacids and anticholinergic agents are important as inhibitors of aggressive factors such as acidpepsin and have been frequently used for the treatment of peptic ulcers from olden times. However, whether or not both agents promote the healing of chronic ulcers still remains uncertain. Therefore, the present study was designed to clarify the effects of dried aluminium hydroxide gel, an antacid. and propantheline bromide, an anticholinergic agent, on the healing of acetic acid ulcer in rats by the method of histological measurements (1).

Gastric ulcer was induced in male SpragueDawley strain rats weighing approx. $180 \mathrm{~g}$ by injection of $0.05 \mathrm{ml}$ of $20 \%$ acetic acid into the serosal layer in the glandular part of the stomach, in accordance with the method described by Takagi et al. (2). Test drugs were suspended (dried aluminium hydroxide gel) or dissolved (propantheline bromide) in $1 \%$ gum arabic and administered orally. twice a day, in a volume of $0.5 \mathrm{ml}$ per $100 \mathrm{~g}$ of body weight for 14 consecutive days after the operation. Control animals were given $1 \%$ gum arabic only instead of test drug. On the 15th day, the animals were sacrificed by rapid decapitation. The stomachs were removed, filled with $5 \mathrm{ml}$ of $10 \%$ formalin and allowed to stand for $5 \mathrm{~min}$. Then, the stomachs were cut open along the greater curvature. The longitudinal and abscissal lengths of the ulcer were measured with a caliper, and the product of both lengths $\left(\mathrm{mm}^{2}\right)$ was expressed in terms of the macroscopic ulcer index. After measuring the ulcer size, the stomach tissues were again immersed in 10\% formalin for $24 \mathrm{hr}$. The formalin-fixed tissues were cut so that a small amount of the normal tissue surrounding the ulcer remained. Thereafter, the central part of the ulcer was cut vertically against the serosa along the long diameter. These tissues cut in half were embedded in paraffin, and the paraffin sections were stained with hematoxylin and eosin and Masson trichrome. Histological measurements were performed under light micrograph of hematoxylin and eosin-stained preparations, as shown in Fig. 1. Of the data measured here, the thickness of the ulcer base and the defective area in the ulcerated region were used for evaluating the effects of test drugs. Moreover, the decreasing index of exposed ulcer floor and the mucosal regeneration index were calculated on the basis of the measured data. In this case, the decreasing index of exposed ulcer floor is taken as 100 when the ulcer floor is completely covered with the regenerated mucosa. 

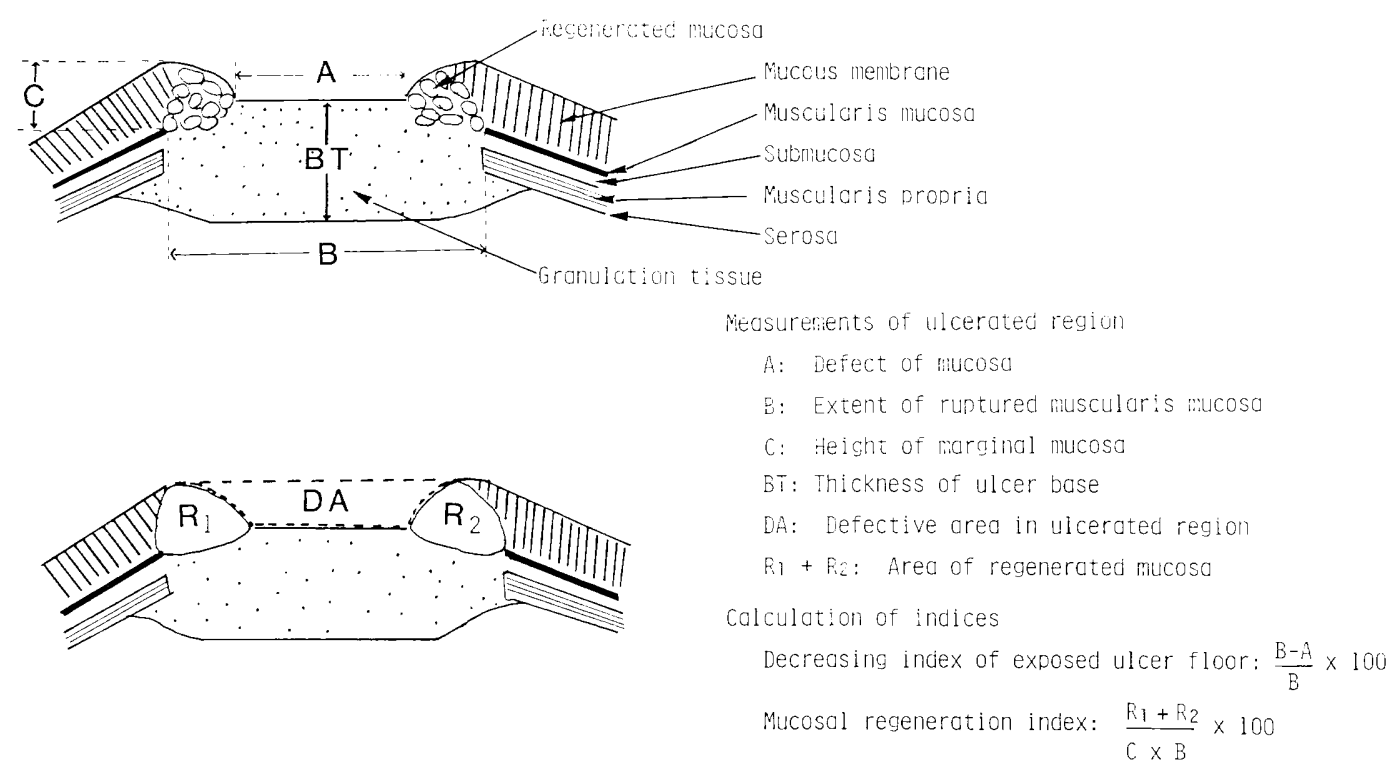

Fig. 1. Method for histological measurements.

On the other hand, the mucosal regeneration index is taken as 100 when the ulcer floor is completely covered with the regenerated mucosa equal to the marginal mucosa of the ulcer in height. Then, the development index of collagen fibers was calculated in accordance with the classification of Tabayashi (3) under light microscopic observation of the Masson trichrome-stained preparation. The data obtained were statistically analyzed by Student's $t$-test.

The results are given in Fig. 2. Dried aluminium hydroxide gel significantly decreased the macroscopic ulcer index and the defective area in the ulcerated region by 42 and $48 \%$, respectively. In addition, this drug significantly increased the decreasing index of exposed ulcer floor and the mucosal regeneration index by approx. $40 \%$. However, this did not affect the thickness of the ulcer base indicating the degree of proliferation of granulation tissue formed in the defective region, and on the development index of collagen fibers. On the other hand, propantheline bromide had little effect on any of the measured parameters.

The macroscopic ulcer index cannot assess the depth of the ulcer and histological changes in the ulcerated region. On the other hand, the method of histological measurements can quantitatively evaluate histological changes such as the regeneration of defective mucosa and the proliferation of granulation tissue formed in the defective region (1). In the present study, it was demonstrated by the method of histological measurements that dried aluminium hydroxide gel remarkably promoted the regeneration of defective mucosa without affecting the formation of granulation tissue in the defective region during the ulcer healing process. It is wellknown that antacids containing aluminium administered orally have an adhesive property to gastric mucosa in addition to an acidneutralizing action. However, the acidneutralizing action of this kind of antacid is very weak in spite of the prolonged action (4). Moreover, in the present experiment, propantheline bromide which is known to inhibit acid secretion did not bring about any 


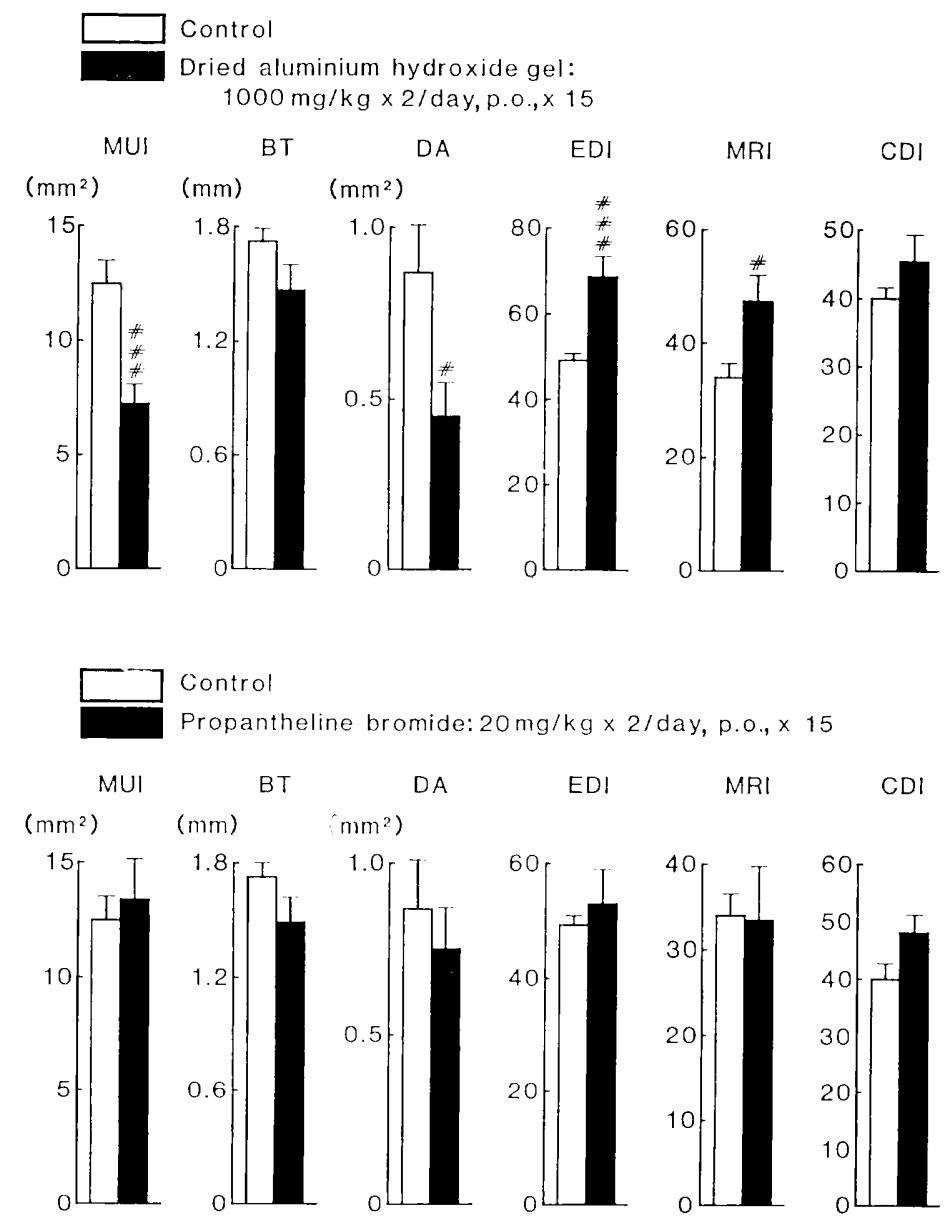

Fig. 2. Effects of dried aluminium hydroxide gel and propantheline bromide on the healing of acetic acid ulcer in rats. Each column denotes the mean value with S.E. $(N=10-11)$. MUI: Macroscopic ulcer index. BT: Thickness of ulcer base. DA: Defective area in ulcerated region. EDI: Decreasing index of exposed ulcer floor. MRI: Mucosal regeneration index. CDI: Development index of collagen fibers. \# $\mathrm{P}<0.05$, 非非 $<0.001$, compared with the control.

beneficial effects. It is unlikely from these findings that dried aluminium hydroxide gel exerts its ulcer healing-promoting effect by acid-neutralizing action. On the other hand, Tanaka et al. (5) reported that there was a good relationship between the antiulcer effect and aluminium content adhered to the gastric wall when aluminium hydroxide or aluminium-containing antiulcer drugs was given to rats with acetic acid ulcer for a week. Therefore, the adhesive property of dried aluminium hydroxide gel may play an important role in the protection of the mucosa from gastric acid, and this may be relevant to its ulcer healing-promoting effect.

\section{References}

1 Ito, M., Fujii, A. and Suzuki, Y.: Long-term histological observation of the healing process of acetic acid ulcer in rats and apparent relapse. In Experimental UIcer-Research Trends in Japan, Edited by Tsuchiya. M., Oda, M. and Okazaki, I., p. 201-206. Yurinsha, Ltd., Tokyo (1983)

2 Takagi, K., Okabe, S. and Saziki, R.: A new method for the production of chronic gastric ulcer in rats and the effect of several drugs on its healing. Japan. J. Pharmacol. 19, 418-426 (1969)

3 Tabayashi, T.: Production of chronic ulcer in 
rats by clamping-cortisone method and histological findings. J. Gastroenterol. 62, 15331547 (1965) (in Japanese)

4 Miyoshi, M. and Kawai, K.: Studies on acidneutralizing action of antacids. J. Kyoto Pref. Univ. Med. 79, 132-136 (1970) (Abs. in Eriglish)
5 Tanaka, H., Kojima, T. and Marumo, H.: Relation between the anti-ulcer effect and the formation of the adhered complex to the mucosa by $\mathrm{N}$ acetyl-L-glutamine aluminum complex (KW110). Pharmacometrics 11, 71-76 (1976) (Abs. in English) 